\title{
Differential diagnosis
}

\section{A standardised sequential clinical examination identified probable causes of syncope in $69 \%$ of patients}

\author{
Sarasin FP, Louis-Simonet M, Carballo D, et al. Prospective evaluation of patients with syncope: a population-based study. \\ Am J Med 2001 Aug 15;111:177-84.

\section{QUESTION: What are the causes of syncope in patients presenting to the emergency department (ED)?}

Design

18 month cohort study.

\section{Setting}

The ED and inpatient services of a major primary and tertiary care hospital in Geneva, Switzerland.

Source of funding: Swiss National Research Foundation.

For correspondence: $\mathrm{Dr}$ F P Sarasin, Hôpital Cantonal, Geneva, Switzerland. francois.sarasin@ houge.ch.

\section{Patients}

788 consecutive patients $\geqslant 18$ years of age who presented to the ED with a chief symptom of syncope. Patients with symptoms clearly compatible with seizure disorders, vertigo, dizziness, coma, or shock were excluded. 650 patients $(82 \%)$ were included in the analysis (mean age $60 \mathrm{y}, 52 \%$ women).

\section{COMMENTARY}

Determining a cause of syncope is often difficult. Previous studies have shown that cause could be assigned in $59 \%$ to $87 \%$ of patients. ${ }^{1-4}$

Vasovagal syncope is common in an ED population (37\% to 40\%), but orthostatic hypotension has only accounted for up to $7.6 \%$ in previous studies. ${ }^{1-3}$ These 2 diagnoses accounted for $61 \%$ of the causes in the study by Sarasin $e t$ al. The reason for the high proportion of patients with orthostatic hypotension is not clear. Older patients have a higher prevalence of orthostatic hypotension, a possible consequence of the physiological effects of aging, comorbidity, and multiple medications. Patients in previous US studies had a mean age of 41 to 44 years, ${ }^{1-3}$ whereas patients in the study by Sarasin $e t$ al had a mean age of 60 years. Thus, the older age of the patients in this study might account for the higher prevalence of orthostatic hypotension.

Many syncope investigators expect that wider use of loop event monitoring, electrophysiological studies (EP), and tilt testing will lead to diagnoses in virtually all patients with syncope. Although the authors reported a final diagnosis in $86 \%$ of patients, the high rate was because of the result of the history and physical examination, not the result of diagnostic tests.

The tilt testing protocol did not use chemical stimulation. In most laboratories this protocol has a relatively low yield. Furthermore, EP testing was only done in 16 patients. It is possible that with chemical stimulation (isoproterenol or nitroglycerine) during tilt testing and wider use of EP testing, many of the remaining patients could have been assigned diagnoses.

This study confirms the central role of careful clinical assessment of patients with syncope. The role of extensive testing with EP studies and tilt testing in the remaining patients requires further study.

Wishwa N Kapoor, MD, MPH University of Pittsburgh Pittsburgh Pennsylvania USA

1 Day SC, Cook EF, Funkenstein $\mathrm{H}$, et al. Evaluation and outcome of emergency room patients with transient loss of consciousness. Am J Med 1982;73:15-23.

2 Kapoor WN. Evaluation and outcome of patients with syncope. Medicine 1990;69:160-75.

3 Martin GJ, Adams SL, Martin HG, et al. Prospective evaluation of syncope. Ann Emerg Med 1984;13:499-504.

4 Linzer M, Yang EH, Estes NA 3rd, et al. Diagnosing syncope. Part 1: Value of history, physical examination, and electrocardiography. Clinical Efficacy Assessment Project of the American College of Physicians. Ann Intern Med 1997;126:989-96.

\section{Diagnostic strategy}

All patients had a standardised evaluation that included a complete history, physical, and neurological examination; laboratory examination (haematocrit and serum concentrations of creatine kinase and glucose); 12 lead electrocardiogram (ECG); testing for orthostatic hypotension; and bilateral carotid massage in patients without contraindications. After the initial evaluation, patients were classified into 3 groups: strongly suspected cause, signs or symptoms suggested a specific cause but required confirmation by selected diagnostic procedures, and undetermined cause. Patients with undetermined causes had extensive cardiovascular testing, transthoracic echocardiography, continuous loop event recorder, signalaveraged ECG, and passive upright tilt testing.

\section{Main outcome measure}

Final diagnosis.

\section{Main results}

After the initial clinical evaluation, a cause of syncope was strongly suspected in 446 patients $(69 \%)$. A cause was suspected but required confirmation by selective diagnostic testing in 67 patients $(10 \%)$; the diagnosis was confirmed in $49(73 \%)$. A specific cause was undetermined in 155 patients (24\%); 122 of these patients had an extensive workup, and probable cause was established in 30 of these patients $(25 \%)$. The final diagnoses are summarised in the table.

\section{Conclusion}

A standardised clinical evaluation provided a probable cause of syncope in $69 \%$ of patients presenting to the emergency department with a chief symptom of syncope.

Final diagnoses in patients presenting with syncope

\begin{tabular}{lc} 
Diagnosis & $\begin{array}{c}\text { Number of } \\
\text { patients }(\%)\end{array}$ \\
Cardiac causes & $69(11)$ \\
\hline Arrhythmias & $44(7)$ \\
\hline Acute coronary syndrome & $9\{1\}^{*}$ \\
\hline Aortic stenosis & $8\{1\}^{*}$ \\
\hline Pulmonary embolism & $8\{1\}^{*}$ \\
\hline Non-cardiac causes & $456(70)$ \\
\hline Vasodepressor syncope & $242(37)$ \\
\hline Orthostatic hypotension & $158(24)$ \\
\hline Carotid sinus hypersensitivity & $6(1)$ \\
\hline Neurological & $30(5)$ \\
\hline Psychiatric & $11(2)$ \\
\hline Other & $9\{1\}^{*}$ \\
\hline Unknown & $92(14)$ \\
\hline${ }^{*}$ Calculated from data in article.
\end{tabular}

